\title{
Using the latest developments in mobile technology to improve students' engagement with assessment feedback
}

\author{
Dr. Carolyne Jacobs \& Michael Wilson \\ Department of Curriculum and Quality Enhancement \\ University of Portsmouth \\ Portsmouth, United Kingdom \\ carolyne.jacobs@port.ac.uk \\ michael.wilson@port.ac.uk
}

\author{
Charles Barker \\ Portsmouth Business School \\ University of Portsmouth \\ Portsmouth, United Kingdom \\ charles.barker@port.ac.uk
}

\begin{abstract}
This presentation focuses on web-based 'app' that brings together feedback for Moodle-based assignments in one location.

The app provides an individual student with summary feedback from tutors and provisional/final marks for all their assignments. The app is available on desktop and mobile devices.
\end{abstract}

This development is based on the hypothesis that by making feedback more accessible, students are more likely to engage with it and this will have a beneficial impact on their learning.

Keywords-assessment, engagement, accessibility, mobile access

\section{INTRODUCTION}

Student satisfaction surveys and colleagues' experience show that students do not readily engage with lecturers' feedback on their assessments. Improving students' engagement with assessment and feedback is a problem faced by many institutions in the higher education sector. Practice within the sector is diverse and issues include: timing and using feedback to enhance learning and development; inconsistencies relating to style and volume; access to feedback for immediate or future reference [1].

Traditionally, providing student feedback involved paperbased activities. Although there is a move towards online marking, in many cases it is still a disjointed process involving staff marking manually and students collecting marked papers from a number of different locations. This is both time consuming and dis-engaging for students and staff.

Over $94 \%$ of the adult population in the UK currently own one or more mobile devices [2]. This high level of ownership offers universities a new channel of communication that can be used to effectively engage students in their learning.

Students' expectations have also changed in recent years, they now expect to be able to access learning materials and receive information from a range of different devices and flexible locations [3]. Using technology to create an integrated assessment cycle that includes online submission, marking and feedback is part of that process. Online submission and in some cases, online marking, is becoming increasingly common in many UK institutions. Online feedback is also becoming more common, but it generally tends to be piecemeal and students generally have to access a range of different locations to view feedback.

Staff and students exposed to separate feedback artefacts can lead to a situation where each piece of feedback is viewed in isolation, which is not helpful in terms of the students' overall learning development [4]. An activity where students are trying to make sense of feedback, often without help, is contrary to the majority of Hamish Coates' [5] five dimensions of student engagement:

1. active and collaborative learning;

2. participation in challenging academic activities;

3. formative communication with academic staff;

4. involvement in enriching educational experiences;

5. feeling legitimated and supported by university learning communities.

The notion of using technology to enhance students' engagement with feedback is endorsed in Parkin, Hepplestone et al. [6]; although the ideas presented were focused on the generation of feedback, the same principle of actively involving students still applies. Price, Handley et al. [7] make the point that students most value the feedback which they can use immediately to improve an assessment mark.

\section{IMPLEMENTATION}

The Technology Enhanced Learning (TEL) teams at the University of Portsmouth have developed a web app that brings together assessment marks and lecturer's feedback for the year's units (modules) in one location. 
The design process involved producing mock-ups of how the web application could display summaries of Moodle, Turnitin and Group assignment feedback and grades. The designs were presented to a focus group consisting of academic staff, technical support staff (on-line course developers) and MA students. The app design was refined based on discussions around the key elements which would be of use to students.

Using the feedback app, students are able to access their feedback on a computer, laptop or mobile device and construct meaning from it and work in collaboration with staff to scaffold future learning. This approach is founded on notions of social constructivism [8] and situated learning [9].

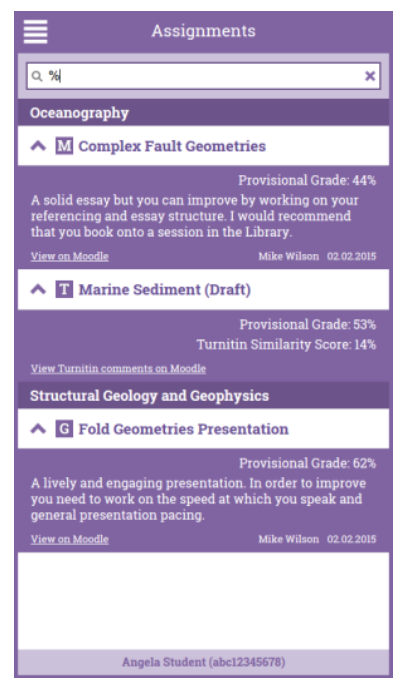

\section{USAGE}

The TEL team raised awareness about the feedback app at the university through presentations, news announcements and teaching and learning committees.

This app went 'live' in March 2015 and was promoted as follows "If you can see your feedback on Moodle, you can see it here." On the first day over 1,500 students accessed the app, but this figure settled down to about 100 students a day with an increase to between 150 and 200 when assessments had been marked by lecturers.

Since launch over 5,500 individual students have logged into MyFeedback to check assessment feedback. Of this number around 1800 students have used MyFeedback for twenty second or more, suggesting that they are reading some or all of the feedback comments. On average, students spend just over a minute checking feedback per visit, although some students spend a significantly longer amount of time using the app.

The distribution of students who accessed the app was similar across the five faculties within the University as Table 1 illustrates.
TABLE I. USAGE BY FACULTY

\begin{tabular}{|l|c|}
\hline \multicolumn{2}{|c|}{ March 2015 - August 2015 } \\
\hline Fcience & $\begin{array}{c}\text { Unique students to } \\
\text { access app }\end{array}$ \\
\hline Humanities & 1279 \\
\hline Technology & 1236 \\
\hline Portsmouth Business School & 1204 \\
\hline Creative and Cultural Industries & 1020 \\
\hline
\end{tabular}

\section{FEEDBACK}

The hypothesis underpinning this development is that allowing students to access feedback for several assessments in one place will help encourage and assimilate feedback as part of the learning process and consequently, improve their learning, levels of achievement and self-regulatory feedback seeking behaviour [10].

Gathering student views about the feedback app helps identify the usefulness of providing this type of functionality in a responsive environment.

Undergraduate and postgraduate students studying for LLB Law, LLM Law, LLM Corporate Governance and Law, BSc Social Work and MSc Forensic Accounting were interviewed about their experiences using the feedback app. The aim of the interviews was to look at the impact that the app has had on the way in which assessment feedback is used by students with particular emphasis on the development of self-regulatory learning behaviour. A theoretical framework based on activity theory [11] [12] was used to explore the mediating role of the app in the assessment feedback process and compare it with paper-based assessment feedback and existing virtual learning environment (VLE) -based assessment feedback systems.

An analysis of the interview data using the activity system framework developed for the research suggests that the app offers a system that the students find easy to use; that encourages tutor/student dialogue and that supports student engagement with their feedback. However, the key finding from the interview data is that the app allows the student to engage with multiple episodes of assessment feedback and so support the development of their own selfregulatory learning behaviour. Behaviour that the students felt was not adequately supported by paper-based or VLE-based feedback.

\section{CONCLUSIONS}

This paper focuses on a pilot development at the University of Portsmouth. Developments are in an early stage and consequently, it is only possible to report preliminary findings. However, early experience and findings suggest that making feedback easily accessible in one location aids engagement and helps students derive meaning from their feedback. 
Feedback from undergraduate and postgraduate students of two faculties indicates that the app also encourages students to take some responsibility, for their learning. This is particularly important at a time when there is increasing number of part-time and distance learners, who may not have access to the university campus or campus-based resources.

The next stage in this project is to promote the wider university use of the feedback app to help engage students in the feedback process and enhance their learning.

\section{ACKNOWLEDGEMENTS}

Michael Wilson, Senior Educational Technologist, Technology Enhanced Learning, University of Portsmouth. Mike developed the app and contributed to this paper.

Charles Barker BA, MA, Barrister, FHEA, Associate Head, School of Law, Portsmouth Business School, University of Portsmouth. Charles conducted a piece of qualitative research in regards to the app with students from two faculties and has also contributed to this paper.

\section{REFERENCES}

The template will number citations consecutively within brackets [1]. The sentence punctuation follows the bracket [2]. Refer simply to the reference number, as in [3] - do not use "Ref. [3]" or "reference [3]" except at the beginning of a sentence: "Reference [3] was the first ..."

Number footnotes separately in superscripts. Place the actual footnote at the bottom of the column in which it was cited. Do not put footnotes in the reference list. Use letters for table footnotes.

Unless there are six authors or more give all authors' names; do not use "et al.". Papers that have not been published, even if they have been submitted for publication, should be cited as "unpublished" [4]. Papers that have been accepted for publication should be cited as "in press" [5]. Capitalize only the first word in a paper title, except for proper nouns and element symbols.

For papers published in translation journals, please give the English citation first, followed by the original foreign-language citation [6].

[1] G. Eason, B. Noble, and I.N. Sneddon, "On certain integrals of Lipschitz-Hankel type involving products of Bessel functions," Phil. Trans. Roy. Soc. London, vol. A247, pp. 529-551, April 1955. (references)

[2] J. Clerk Maxwell, A Treatise on Electricity and Magnetism, 3rd ed., vol. 2. Oxford: Clarendon, 1892, pp.68-73.

[3] I.S. Jacobs and C.P. Bean, "Fine particles, thin films and exchange anisotropy," in Magnetism, vol. III, G.T. Rado and H. Suhl, Eds. New York: Academic, 1963, pp. 271-350.

[4] K. Elissa, "Title of paper if known," unpublished.

[5] R. Nicole, "Title of paper with only first word capitalized," J. Name Stand. Abbrev., in press.
[6] Y. Yorozu, M. Hirano, K. Oka, and Y. Tagawa, "Electron spectroscopy studies on magneto-optical media and plastic substrate interface," IEEE Transl. J. Magn. Japan, vol. 2, pp. 740-741, August 1987 [Digests 9th Annual Conf. Magnetics Japan, p. 301, 1982].

[7] M. Young, The Technical Writer's Handbook. Mill Valley, CA: University Science, 1989. 